\title{
Fast Computation of the Dyadic Green's Function for Layered Media via Interpolation
}

\author{
Phillip Russell Atkins and Weng Cho Chew, Fellow, IEEE
}

\begin{abstract}
The use of a dyadic layered-medium Green's function as the kernel in a method of moments (MoM) modeling problem greatly reduces the complexity of modeling a stratified medium. Compared to the free-space Green's function, there is an additional cost of having to compute a semi-infinite Sommerfeld integral for each call to calculate the dyadic layered-medium Green's function. This letter discusses a method to tabulate and interpolate the Green's function as a method of reducing the impedance matrix filling time. This method can be used in conjunction with existing methods for increasing the computational speed of the Green's functions.
\end{abstract}

Index Terms-Galerkin method, Green's function, inhomogeneous media, interpolation.

\section{INTRODUCTION}

C OMPUTATIONAL electromagnetics has evolved a myriad of different techniques to be used in order to model the behavior of complex structures, one of the more widely used being the method of moments (MoM) [1]. The formulation for the integral equations used in the MoM varies, but centers around the evaluation of the Green's function. When the modeling problem includes an infinite layered medium, the Green's function can account for the layering in the form of a dyadic Green's function for layered medium (DGLM). The evaluation of the DGLM is costly, as it involves the computation of semi-infinite Sommerfeld integrals. Various techniques have been used in the past for decreasing the computation time of these integrals such as steepest descent [2] and discrete complex images [3], [4]. This letter, however, will evaluate the DGLM using interpolation techniques in order to decrease the overall matrix filling time for the MoM. While this technique has been used in many previous layered-medium problems, previous works have not presented a comprehensive discussion on the implementation, considerations, and results of interpolation for a generalized multilayered problem.

Some works have only focused upon specific cases for interpolation. In [5] and [6], interpolation was applied for the case of planar structures. In papers discussing more general applications, like [7] and [8], the case of three-dimensional (3D) scatterers that penetrate an interface are confined to a

Manuscript received March 23, 2010; accepted April 07, 2010. Date of publication May 10, 2010; date of current version June 07, 2010. This work was supported in part by HKU Research Services, AFOSR Grant AFOSR F9550-04-10326, and the YT Lo Endowed Professor Fund.

The authors are with the Center for Computational Electromagnetics and Electromagnetics Laboratory, Department of Electrical and Computer Engineering, University of Illinois at Urbana-Champaign, Urbana, IL 61801 USA, on leave from the Faculty of Engineering, University of Hong Kong, Pokfulam, Hong Kong (e-mail: phatkins@hku.hk).

Digital Object Identifier 10.1109/LAWP.2010.2050130 half-space problem, ignoring the additional terms that arise when embedded between layers. Other papers only deal with the interpolation in a cursory manner [9], [10] or lack a highly customizable scheme [11]. This letter will deal with a generalized interpolation scheme for planar and 3D scatterers that lie in a multilayered medium. We will briefly discuss an interpolation scheme used for a matrix-friendly formulation [12], while saving details such as error control considerations for a more comprehensive report [13]. Our implementation differs from previous ones in that we have reduced the inner products for the impedance matrix into integrals dependent upon two Sommerfeld integrals and their derivatives. Instead of calculating, storing, and interpolating the terms of the DGLM itself, we will only store these two basic Sommerfeld integrals and interpolate their values and their derivatives and use them to evaluate the DGLM.

\section{Dyadic GREen's Function For LAYERED MEDIA}

The interpolation technique is formulated based upon a previously proposed matrix-friendly formulation [12]. The resulting DGLM is formulated as

$$
\begin{aligned}
\overline{\mathbf{G}}\left(\mathbf{r}, \mathbf{r}^{\prime}\right)= & (\nabla \times \hat{z})\left(\nabla^{\prime} \times \hat{z}\right) g^{\mathrm{TE}}\left(\mathbf{r}, \mathbf{r}^{\prime}\right)+\frac{1}{k_{n m}^{2}}(\nabla \times \nabla \times \hat{z}) \\
& \times\left(\nabla^{\prime} \times \nabla^{\prime} \times \hat{z}\right) g^{\mathrm{TM}}\left(\mathbf{r}, \mathbf{r}^{\prime}\right)\left|z-z^{\prime}\right|>0
\end{aligned}
$$

where $k_{n m}^{2}=\omega^{2} \epsilon_{n} \mu_{m}, m$ is the source layer and $n$ is the observation layer. We will define $k_{m z}=\sqrt{k_{m}^{2}-k_{\rho}^{2}}$. The Green's function terms $g^{\mathrm{TE}}\left(\mathbf{r}, \mathbf{r}^{\prime}\right)$ and $g^{\mathrm{TM}}\left(\mathbf{r}, \mathbf{r}^{\prime}\right)$, respectively relating to the transverse electric (TE) and transverse magnetic (TM) modes, can be expressed in cylindrical coordinates.

$$
\begin{aligned}
& g^{\mathrm{TE}}\left(\mathbf{r}, \mathbf{r}^{\prime}\right)=\frac{i}{4 \pi} \int_{0}^{\infty} \frac{d k_{\rho}}{k_{m z} k_{\rho}} J_{0}\left(k_{\rho} r_{s}\right) F^{\mathrm{TE}}\left(k_{\rho}, z, z^{\prime}\right) \\
& g^{\mathrm{TM}}\left(\mathbf{r}, \mathbf{r}^{\prime}\right)=\frac{i}{4 \pi} \int_{0}^{\infty} \frac{d k_{\rho}}{k_{m z} k_{\rho}} J_{0}\left(k_{\rho} r_{s}\right) F^{\mathrm{TM}}\left(k_{\rho}, z, z^{\prime}\right)
\end{aligned}
$$

where $r_{s}=\sqrt{\left(x-x^{\prime}\right)^{2}+\left(y-y^{\prime}\right)^{2}}$. The function $F$ describes the response of a point source potential embedded in the layered medium [14], [15]. The pole existing at $k_{\rho}=0$ in the integrand is fictitious, and it can be shown that it would be canceled out in the terms described in (6)-(10). In solving a MoM problem, the resulting impedance matrix problem becomes

$$
\begin{aligned}
Z_{i j} & =i \omega \mu_{m}\left\langle\mathbf{J}_{T i}(\mathbf{r}), \overline{\mathbf{G}}\left(\mathbf{r}, \mathbf{r}^{\prime}\right), \mathbf{J}_{j}\left(\mathbf{r}^{\prime}\right)\right\rangle \\
& =i \omega \mu_{m} \int d \mathbf{r} \mathbf{J}_{T i}(\mathbf{r}) \cdot \int d \mathbf{r}^{\prime} \overline{\mathbf{G}}\left(\mathbf{r}, \mathbf{r}^{\prime}\right) \cdot \mathbf{J}_{j}\left(\mathbf{r}^{\prime}\right) .
\end{aligned}
$$


Expanding out the inner product and shifting around the derivatives

$$
\begin{aligned}
&\left\langle\mathbf{J}_{T i}(\mathbf{r}), \overline{\mathbf{G}}\left(\mathbf{r}, \mathbf{r}^{\prime}\right), \mathbf{J}_{j}\left(\mathbf{r}^{\prime}\right)\right\rangle \\
&=\left\langle\nabla \cdot \mathbf{J}_{T i}(\mathbf{r}), g_{\phi}\left(\mathbf{r}, \mathbf{r}^{\prime}\right), \nabla^{\prime} \cdot \mathbf{J}_{j}\left(\mathbf{r}^{\prime}\right)\right\rangle \\
&-\left\langle\mathbf{J}_{T i}(\mathbf{r}) \cdot \hat{z}, g_{z 1}\left(\mathbf{r}, \mathbf{r}^{\prime}\right), \nabla^{\prime} \cdot \mathbf{J}_{j}\left(\mathbf{r}^{\prime}\right)\right\rangle \\
&-\left\langle\nabla \cdot \mathbf{J}_{T i}(\mathbf{r}), g_{z 2}\left(\mathbf{r}, \mathbf{r}^{\prime}\right), \hat{z} \cdot \mathbf{J}_{j}\left(\mathbf{r}^{\prime}\right)\right\rangle \\
&+\left\langle\hat{z} \cdot \mathbf{J}_{T i}(\mathbf{r}), g_{z z}\left(\mathbf{r}, \mathbf{r}^{\prime}\right), \hat{z} \cdot \mathbf{J}_{j}\left(\mathbf{r}^{\prime}\right)\right\rangle \\
&+\left\langle\mathbf{J}_{T S i}(\mathbf{r}), g_{s s}\left(\mathbf{r}, \mathbf{r}^{\prime}\right), \mathbf{J}_{S j}\left(\mathbf{r}^{\prime}\right)\right\rangle
\end{aligned}
$$

with the terms defined as follows:

$$
\begin{aligned}
& g_{s s}\left(\mathbf{r}, \mathbf{r}^{\prime}\right)=\left(k_{n}^{2}+\partial_{z}^{2}\right) g^{\mathrm{TE}}\left(\mathbf{r}, \mathbf{r}^{\prime}\right) \\
& g_{z z}\left(\mathbf{r}, \mathbf{r}^{\prime}\right)=k_{n m}^{2} g^{\mathrm{TM}}\left(\mathbf{r}, \mathbf{r}^{\prime}\right)-\partial_{z} \partial_{z^{\prime}} g^{\mathrm{TE}}\left(\mathbf{r}, \mathbf{r}^{\prime}\right) \\
& g_{z 1}\left(\mathbf{r}, \mathbf{r}^{\prime}\right)=\frac{\mu_{n}}{\mu_{m}} \partial_{z^{\prime}} g^{\mathrm{TM}}\left(\mathbf{r}, \mathbf{r}^{\prime}\right)+\partial_{z} g^{\mathrm{TE}}\left(\mathbf{r}, \mathbf{r}^{\prime}\right) \\
& g_{z 2}\left(\mathbf{r}, \mathbf{r}^{\prime}\right)=\frac{\epsilon_{n}}{\epsilon_{m}} \partial_{z} g^{\mathrm{TM}}\left(\mathbf{r}, \mathbf{r}^{\prime}\right)+\partial_{z^{\prime}} g^{\mathrm{TE}}\left(\mathbf{r}, \mathbf{r}^{\prime}\right) \\
& g_{\phi}\left(\mathbf{r}, \mathbf{r}^{\prime}\right)=\frac{\partial_{z} \partial_{z^{\prime}}}{k_{n m}^{2}} g^{\mathrm{TM}}\left(\mathbf{r}, \mathbf{r}^{\prime}\right)-g^{\mathrm{TE}}\left(\mathbf{r}, \mathbf{r}^{\prime}\right) .
\end{aligned}
$$

Thus, the DGLM that is used for the calculation of the impedance matrix for the electric field integral equation can be decomposed into the summation of $g^{\mathrm{TE}}, g^{\mathrm{TM}}$, and their derivatives with respect to $z$ and $z^{\prime}$. In addition, by taking into consideration special cases, $g^{\mathrm{TE}}$ and $g^{\mathrm{TM}}$ can be made dependent upon one or two independent variables, allowing for faster and more accurate interpolation. When the source and observation points lie in the same layer, that is $n=m$, the function $F$ can be decomposed into the summation of two functions as follows:

$$
\begin{aligned}
F\left(k_{\rho}, z, z^{\prime}\right)= & F_{1}\left(r_{s},\left|z-z^{\prime}\right|\right)+F_{2}\left(r_{s}, z+z^{\prime}\right) \\
F_{1}\left(k_{\rho},\left|z-z^{\prime}\right|\right)= & e^{i k_{m z}\left|z-z^{\prime}\right|}+2 \cos \left(k_{m z}\left|z-z^{\prime}\right|\right) \\
& \cdot e^{2 i k_{m z}\left(d_{m}-d_{m-1}\right)} \tilde{R}_{m, m-1} \tilde{R}_{m, m+1} \widetilde{M}_{m} \\
F_{2}\left(k_{\rho}, z+z^{\prime}\right)= & {\left[e^{-i k_{m z}\left(z+z^{\prime}+2 d_{m-1}\right)} \tilde{R}_{m, m-1}\right.} \\
& \left.+e^{i k_{m z}\left(z+z^{\prime}+2 d_{m}\right)} \tilde{R}_{m, m+1}\right] \widetilde{M}_{m} .
\end{aligned}
$$

Here, $\widetilde{R}$ represents the generalized reflection coefficients. The function $F_{1}$ contains the direct contribution from the source, and this contribution can be ignored since it can be calculated in closed form. We will henceforth refer to $g$, whether TE or TM, as $g=g_{1}+g_{2}$, where $g_{1}$ is the integral containing $F_{1}$ and $g_{2}$ is the integral containing $F_{2}$. Specifically

$$
\begin{aligned}
g_{1}\left(\mathbf{r}, \mathbf{r}^{\prime}\right)= & \frac{i}{2 \pi} \int_{0}^{\infty} \frac{d k_{\rho}}{k_{m z} k_{\rho}} J_{0}\left(k_{\rho} r_{s}\right) \cos \left(k_{m z}\left|z-z^{\prime}\right|\right) \\
& \times e^{2 i k_{m z}\left(d_{m}-d_{m-1}\right)} \tilde{R}_{m, m-1} \tilde{R}_{m, m+1} \widetilde{M}_{m} \\
g_{2}\left(\mathbf{r}, \mathbf{r}^{\prime}\right)= & \frac{i}{4 \pi} \int_{0}^{\infty} \frac{d k_{\rho}}{k_{m z} k_{\rho}} J_{0}\left(k_{\rho} r_{s}\right) \\
& \times\left[e^{-i k_{m z}\left(z+z^{\prime}+2 d_{m-1}\right)} \tilde{R}_{m, m-1}\right. \\
& \left.\quad+e^{i k_{m z}\left(z+z^{\prime}+2 d_{m}\right)} \tilde{R}_{m, m+1}\right] \widetilde{M}_{m} .
\end{aligned}
$$

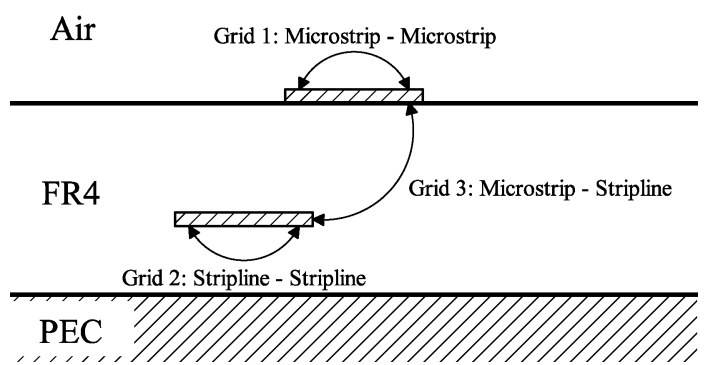

Fig. 1. Three 1D grids that contain the interactions of the scatterers in a typical layered-medium problem.

To facilitate the fast evaluation of the above integrals, we incorporated singularity-subtraction of the pole at $k_{\rho}=0$ and quasi-static subtraction to promote fast convergence of the integrand [13]. In addition, the quasi-static subtraction was necessary to improve the interpolation error by smoothing out the resulting Green's functions.

\section{TABULATION AND INTERPOLATION SCHEME}

The Green's function terms $g^{\mathrm{TE}}$ and $g^{\mathrm{TM}}$ are dependent upon the spatial position of the source and observation points $r_{s}, z$, and $z^{\prime}$. Normally, the two functions require a 3D interpolation and tabulation due to the separation of the three variables. This separation is seen when we take into consideration the function $F\left(z, z^{\prime}\right)$ when used in (2) and (3). As shown above, however, when the source and observation points are located in the same layer, the interpolation and tabulation can be defined in one or two dimensions by splitting $g^{\mathrm{TE}, \mathrm{TM}}$ into the summation of the functions $g_{1}^{\mathrm{TE}, \mathrm{TM}}\left(r_{s},\left|z-z^{\prime}\right|\right)$ and $g_{2}^{\mathrm{TE}, \mathrm{TM}}\left(r_{s}, z+z^{\prime}\right)$.

The proposed tabulation scheme is to precompute over a grid space all combinations of possible source and observation points that encompass the physical volume of the scatterer. When the source and observation points lie across different layers of inhomegeneity, the values of $g^{\mathrm{TE}, \mathrm{TM}}$ will need to be calculated using a 3D interpolation. When the source and observation points lie in the same layer, then they are mapped onto two-dimensional (2D) grids for the interpolation of $g_{1}^{\mathrm{TE}, \mathrm{TM}}$ and $g_{2}^{\mathrm{TE}, \mathrm{TM}}$.

Problems where the scatterer lies solely in one or more planes parallel to the layer boundaries allow us to use one-dimensional (1D) grids and interpolation. For example, one would need three 1D grids to model a microstrip line and a stripline. As shown in Fig. 1, we assume a three-layered medium of air, FR4, and copper cladding. The microstrip and stripline are assumed to be $2 \mathrm{D}$ strips confined to the vertical displacements of $z_{1}$ and $z_{2}$. Thus, one grid will contain all the source and observation points for the interaction of currents on the microstrip line itself, another grid will be the interactions within the stripline, and the final grid will contain the interactions between the microstrip and stripline. These correspond to the possible combinations of $z+z^{\prime}$ and $\left|z-z^{\prime}\right|$.

The actual interpolation was performed using Lagrangian polynomial interpolation with the derivatives estimated by taking the derivative of the Lagrangian coefficients. A naive approach to this would require that the interpolations be $O\left(N^{2}\right)$ or $O\left(N^{3}\right)$, but this can be avoided by careful formulation so 
TABLE I

(a) RCS RESUlTS AND (b) COMPARISON OF RUNTIMES FOR VARIOUS PEC SPHERE SiMULATIONS

\begin{tabular}{c|c|c}
\hline Case & Direct Result $(\phi, \theta)$ & Interpolation Result $(\phi, \theta)$ \\
\hline \hline Above & $(-0.279545,-53.4252) \mathrm{dBsm}$ & $(-0.278557,-53.4215) \mathrm{dBsm}$ \\
Embedded & $(0.121033,-49.5591) \mathrm{dBsm}$ & $(0.12051,-49.5636) \mathrm{dBsm}$ \\
Below & $(-9.30028,-74.4569) \mathrm{dBsm}$ & $(-9.30136,-74.5169) \mathrm{dBsm}$ \\
\hline
\end{tabular}

(a)

\begin{tabular}{c|c|c|c}
\hline Case & Direct Time & Interpolation Time & Tabulation Time \\
\hline \hline Above & $22 \mathrm{~min} 14.07 \mathrm{sec}$ & $1 \mathrm{~min} 23.22 \mathrm{sec}$ & $2.18 \mathrm{sec}$ \\
Embedded & $70 \mathrm{~min} 53.72 \mathrm{sec}$ & $2 \mathrm{~min} 45.52 \mathrm{sec}$ & $36.59 \mathrm{sec}$ \\
Below & $30 \mathrm{~min} 1.40 \mathrm{sec}$ & $1 \mathrm{~min} 29.27 \mathrm{sec}$ & $2.66 \mathrm{sec}$ \\
\hline
\end{tabular}

(b)

TABLE II

(a) RCS Results AND (b) COMPARIson of Runtimes For the Two Circular Patches

\begin{tabular}{c|c|c}
\hline Frequency & Direct Result $(\phi, \theta)$ & Interpolation Result $(\phi, \theta)$ \\
\hline \hline $7.0 \mathrm{GHz}$ & $(-43.0374,-111.600) \mathrm{dBsm}$ & $(-43.0475,-111.602) \mathrm{dBsm}$ \\
$15.0 \mathrm{GHz}$ & $(-46.1329,-89.2315) \mathrm{dBsm}$ & $(-46.1507,-89.1973) \mathrm{dBsm}$ \\
\hline
\end{tabular}

(a)

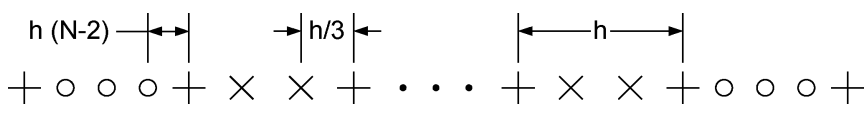

Fig. 2. Example of the sampling of the tabulation grid along one dimension. Here, $N=6$.

that the interpolation of the derivatives will be $O(N)$. However, because we cannot interpolate across the boundaries of the tabulation grids, there exist problems interpolating near the boundaries. This is because the data points are shifted so that they do not symmetrically surround the desired point due to the impassable boundary, thus increasing the error in the interpolation [16]. To counteract this, we can create a finer mesh for the tabulation grid and only run the mesh along the boundaries, allowing for a coarser grid on the interior. An acceptable method was found by dividing the region between the boundary point and the first point on the interior such that the number of points added would be equal to half the number of points used in the interpolating polynomial. Furthermore, two additional points were added between the second and third coarse points.

An example of the sampling of the tabulation grid along a single dimension can be seen in Fig. 2, where we are assuming that the number of points used for the interpolating polynomial is $N=6$. The vertical crosses represent the coarse grid points. The circles represent the fine grid created along the boundary, while the X's were placed in between the second and third coarse grid points to decrease the maximum error in that region. By spacing the grid points in terms of wavelength, it was found that the performance of the interpolation remains consistent across varying frequencies. This invariance is due to the fact that the spatial dimensions are scaled by the wavenumber in the DGLM integrals.

\section{VALIDATION AND RESULTS}

The validity of the original DGLM has been previously presented, and the interpolation and tabulation method was checked against the results of directly integrating the DGLM as in [12]. A comparison was made in the bistatic radar cross section (RCS) produced by the scattering off a perfectly electrically conducting (PEC) sphere constructed with 420 edges. This was done with the sphere located completely above a half-space, embedded in the middle of a three-layer medium and placed in the bottom

\begin{tabular}{c|c|c|c}
\hline Frequency & Direct Time & Interpolation Time & Tabulation Time \\
\hline \hline $7.0 \mathrm{GHz}$ & $191 \mathrm{~min} 32.63 \mathrm{sec}$ & $32.84 \mathrm{sec}$ & $1.72 \mathrm{sec}$ \\
$15.0 \mathrm{GHz}$ & $145 \mathrm{~min} 19.64 \mathrm{sec}$ & $39.75 \mathrm{sec}$ & $2.16 \mathrm{sec}$ \\
\hline
\end{tabular}

(b)

layer of a three-layer geometry. The direct integration results were calculated using an adaptive Simpson's integration routine, while an adaptive quadrature routine from QUADPACK [17] was used to calculate the values in the tabulation grids for the interpolation. The tabulation grids used six points for the interpolating polynomials and a coarse grid spacing of $\lambda / 25$.

The results, generated on a laptop equipped with a $2.53-\mathrm{GHz}$ Intel Core2 Duo processor, can be seen in Table I(a) and I(b). The reported times to fill the impedance matrix demonstrate an appreciable speedup, on the order of a factor of 10, when the scatterer is confined to the top or bottom layer. Placing the sphere in an embedded layer increases the interpolation and tabulation time because an additional set of integrals, $g_{1}$, needs to be tabulated and interpolated.

Another set of tests were run to verify the use of 1D interpolations using planar structures. Here, two circular PEC patches of 272 interior edges each were placed on top of and inside of the middle layer. This allows the interpolation code to create three sets of tabulation grids using 1D interpolations. The results are shown in Table II(a) and II(b). Once again the interpolation results compare very favorably with the direct integration, but have an appreciable speedup on the order of 100 .

Finally, a comparison was made with interpolating the original five Green's function terms. The original formulation only used quasi-static subtraction for the TM term in $g_{\phi}$ as that was the only term that allowed for a subtraction of a Sommerfeld integral. For the PEC sphere case, the tabulation times were 1.7, 2.3 , and $27.4 \mathrm{~s}$ for the above, below, and embedded case, while the interpolation times were 63,64 , and $106 \mathrm{~s}$. The interpolation times were quicker for the original formulation as it does not require the interpolation of derivatives and it uses slightly fewer interpolations. In addition, under some circumstances, the tabulation time of the five terms was faster as well, which can be accounted for by the fact that cancellation occurs between terms that aid convergence. However, because the quasi-static subtraction was not uniformly applied to all of the five terms as in the newer formulation, the tabulation time suffers greatly when tabulating near boundaries. This can be seen in Fig. 3, where we compare the tabulation times when we place the PEC sphere in the embedded layer and vary the gap distance between the sphere and the adjacent boundaries. The tabulation time for the original formulation increases exponentially as the tabulation 


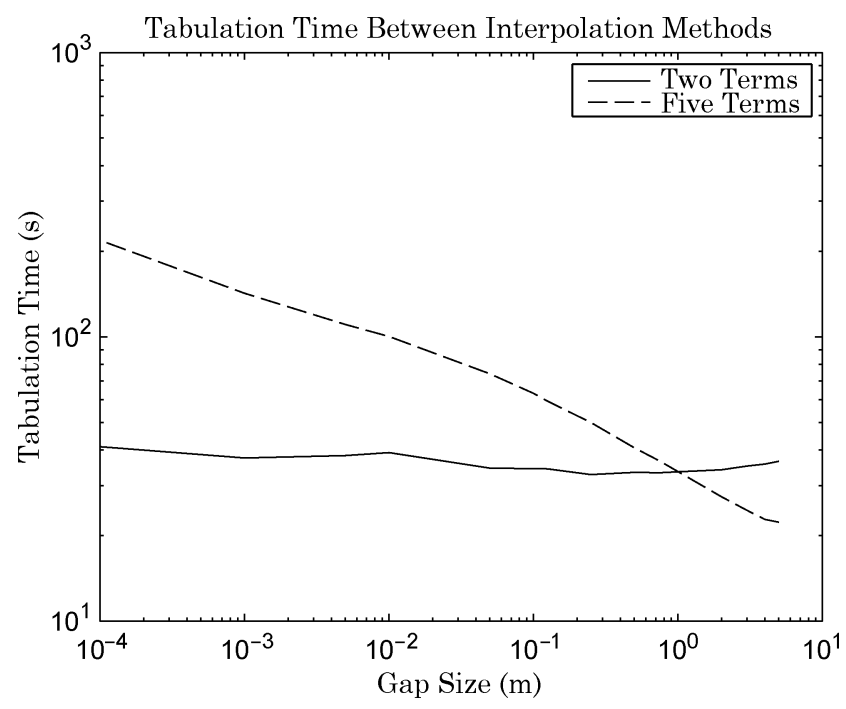

Fig. 3. Comparison between the tabulation times for the proposed and original five-term interpolations.

grids move to encompass points closer to the boundaries. The accuracy of the interpolation for near-field terms also suffers due to the presence of the weak singularity. The new quasi-static subtraction technique prevents such penalties from occurring. We can see that the tabulation time remains fairly constant in Fig. 3, and the quasi-static subtraction has been shown to improve the error resulting from the interpolation.

\section{CONCLUSION}

While interpolation has been used previously in many layered-medium MoM problems, this letter presents a new implementation. The DGLM used has five Green's function terms, shown in (6)-(10), that need to be evaluated for each inner product for each impedance matrix entry. However, at the cost of one or two additional interpolations, our method only requires the storage and evaluation of two integrals instead of five. This comes at the cost of having to increase the density of the tabulation grids in order to compensate for the decreased accuracy of interpolating the derivatives and the effects of cancellation. Despite this, our formulation allows us to have a predictable matrix filling time that is much faster than traditional integration and is still computationally comparable to traditional interpolation. In addition, our new quasi-static subtraction technique allows us to have better accuracy and tabulation times over the direct interpolation of the five terms.

The speed up obtained by the interpolation varies due to the fact that the integration time is dependent upon the geometry of the background layers and the scatterer. However, we experience vast improvements for using one- or two-dimensional interpolation in planar structures and structures confined to a single layer. This makes the method ideal for 1D or 2D interpolations where the quasi-static image terms are prominent. This can apply to thin-layered problems or situations where objects penetrate the boundaries between layers. For any 3D interpolations, the more traditional interpolations can be used at a slight cost of increased memory. This can be done independent of the impedance matrix entries for interactions within the same layer, which can be handled as discussed in this letter.

\section{REFERENCES}

[1] R. F. Harrington, Field Computation by Moment Methods. New York: IEEE Press, 1993.

[2] T. J. Cui and W. C. Chew, "Modeling of arbitrary wire antennas above ground," IEEE Trans. Geosci. Remote Sens., vol. 38, no. 1, pp. 357-365, Jan. 2000.

[3] Y. L. Chow, J. J. Yang, D. G. Fang, and G. E. Howard, "A closed-form spatial Green's function for the thick microstrip substrate," IEEE Trans. Microw. Theory Tech., vol. 39, no. 3, pp. 588-592, Mar. 1991.

[4] M. I. Aksun, "A robust approach for the derivation of closed-form Green's functions," IEEE Trans. Microw. Theory Tech., vol. 44, no. 5, pp. 651-658, May 1996.

[5] F. Alonso-Monferrer, A. A. Kishk, and A. W. Glisson, "Green's functions analysis of planar circuits in a two-layer grounded medium," IEEE Trans. Antennas Propag., vol. 40, no. 6, pp. 690-696, Jun. 1992.

[6] F. Ling and J.-M. Jin, Full-Wave Analysis of Multilayer Microstrip Problems, ser. Fast and Efficient Algorithms in Computational Electromagnetics. Boston, MA: Artech House, 2001, pp. 729-780.

[7] G. J. Burke and E. K. Miller, "Modeling antennas near to and penetrating a lossy interface," IEEE Trans. Antennas Propag., vol. AP-32, no. 10, pp. 1040-1049, Oct. 1984.

[8] L. Xu, Z. Nie, J. Hu, and J. Wang, "Spatial interpolation method for solution of electromagnetic scattering from objects located in half-space," in Proc. 4th Int. Conf. Microw. Millim. Wave Technol., 2004, pp. 167-170.

[9] J. Chen, A. A. Kishk, and A. W. Glisson, "A 3D interpolation model for the calculation of the Sommerfeld integrals to analyze dielectric resonators in a multilayered medium," Electromagnetics, vol. 20, pp. $1-15,2000$.

[10] K. A. Michalski and J. R. Mosig, "Multilayered media Green's functions in integral equation formulations," IEEE Trans. Antennas Propag., vol. 45, no. 3, pp. 508-519, Mar. 1997.

[11] P. E. Wannamaker, G. W. Hohmann, and W. A. SanFilipo, "Electromagnetic modeling of three-dimensional bodies in layered earths using integral equations," Geophysics, vol. 49, no. 1, pp. 60-74, 1984.

[12] W. C. Chew, J. L. Xiong, and M. A. Saville, "A matrix-friendly formulation of layered medium Green's function," IEEE Antennas Wireless Propag. Lett., vol. 5, pp. 490-494, 2006.

[13] P. R. Atkins and W. C. Chew, Tabulation and Interpolation of the Dyadic Green's Function for Layered Media Univ. Illinois, Urbana-Champaign, Res. Rep. No. CCEML 3-09, Nov. 2009.

[14] W. C. Chew, Waves and Fields in Inhomogeneous Media. New York: IEEE Press, 1995.

[15] W. C. Chew and S. Chen, "Response of a point source embedded in a layered medium," IEEE Antennas Wireless Propag. Lett., vol. 2, pp. 254-258, 2003.

[16] W. Cheney and D. Kincaid, Numerical Mathematics and Computing. Pacific Grove, CA: Ostedt, 1999.

[17] R. Piessens, E. D. Doncker-Kapenga, and C. W. Überhuber, $Q U A D$ PACK: A Subroutine Package for Automatic Integration. New York: Springer, 1983. 